\title{
Electronic structure of a thermoelectric material: BiCuSO
}

\author{
D. Pei, ${ }^{1, *}$ Y.-Y. Lv, ${ }^{2,}{ }^{*}$ Y. Y. Y. Xia,${ }^{3}$ Y. W. Li, ${ }^{1,3}$ J. Y. Liu $\odot,{ }^{1}$ N. B. M. Schröter, ${ }^{4}$ C. Cacho, ${ }^{5}$ \\ Z. K. Liu, ${ }^{3}$ L. X. Yang,,${ }^{6,7}$ G. Li, ${ }^{3}$ Y. B. Chen, ${ }^{2}$ and Y. L. Chen ${ }^{1,3,6, \dagger}$ \\ ${ }^{1}$ Department of Physics, University of Oxford, Oxford OX1 3PU, England, United Kingdom \\ ${ }^{2}$ National Laboratory of Solid State Microstructures and Department of Physics, Nanjing University, Nanjing 210093, China \\ ${ }^{3}$ School of Physical Science and Technology, ShanghaiTech University, Shanghai 201210, China \\ ${ }^{4}$ Swiss Light Source, Paul Scherrer Institute, Villigen, Switzerland \\ ${ }^{5}$ Diamond Light Source, Harwell Campus, Didcot OX11 ODE, England, United Kingdom \\ ${ }^{6}$ State Key Laboratory of Low Dimensional Quantum Physics, Department of Physics, Tsinghua University, Beijing 100084, China \\ ${ }^{7}$ Frontier Science Center for Quantum Information, Beijing 100084, China
}

(Received 4 February 2021; revised 22 April 2021; accepted 26 May 2021; published 14 June 2021)

\begin{abstract}
We investigate transport properties and the electronic structure of $\mathrm{BiCuSO}$, a thermoelectric material which was predicted as a possible parent compound for unconventional superconductivity. For the p-type BiCuSO samples studied in this paper, our transport measurements show metallic behavior down to $2 \mathrm{~K}$, and we observe an increase of saturated magnetic moment around $4 \mathrm{~K}$, which could be due to the reorientation of the magnetic easy axis. Using angle-resolved photoemission spectroscopy measurements, we acquired the comprehensive electronic structure of BiCuSO including the predicted flat band, and by carrying out photon polarization dependent measurements we further investigated the orbital characters of bands near the Fermi level. Compared with its sister compound, $\mathrm{BiCuSeO}$, we find the flat band could be the origin of high figure of merit $(Z T)$ value in the $\mathrm{BiCuChO}(C h=\mathrm{S}, \mathrm{Se})$ family.
\end{abstract}

DOI: 10.1103/PhysRevB.103.245121

\section{INTRODUCTION}

Recently, BiCuSO and its sister compound $\mathrm{BiCuSeO}$ have emerged as promising thermoelectric (TE) materials due to their intrinsically low lattice thermal conductivity [1-3]. By $p$-type doping, such as introducing $\mathrm{Cu}$ deficiency [4] or replacing $\mathrm{Bi}^{3+}$ with $A^{2+}(A=\mathrm{Sr}, \mathrm{Pb}, \mathrm{Mg} \ldots)$ [5-11], the TE performance of $\mathrm{BiCuChO}(\mathrm{Ch}=\mathrm{S}, \mathrm{Se})$ can be dramatically enhanced while the lattice structure is barely influenced, which suggests the excellent $\mathrm{TE}$ performance in $\mathrm{BiCuChO}$ is likely rooted in their electronic structures, as is also supported by first-principles calculation $[8,12]$. On the other hand, BiCuSO has attracted research interest due to its potential for hosting unconventional superconductivity [13-18]: Ubaldini et al. reported a superconducting transition at $5.8 \mathrm{~K}$ in polycrystalline $\mathrm{BiCu}_{0.9} \mathrm{SO}$ [13], and Mazin and coworkers proposed that the superconductivity in $\mathrm{BiCu}_{0.9} \mathrm{SO}$ may be mediated by spin fluctuation induced by $\mathrm{Cu}$ deficiency $[14,16]$, despite the controversial experimental observation of semiconductorlike behavior in subsequent transport measurements [17-23]. With these motivations, a systematic investigation on the electronic structure of BiCuSO is needed.

In this paper, millimeter-sized high-quality $\mathrm{BiCuSO}$ single crystals ( $p$ doped due to $\mathrm{Bi}$ and/or $\mathrm{Cu}$ deficiencies) were synthesized for electrical magnetic property measurements and angle-resolved photoemission spectroscopy (ARPES) study.

\footnotetext{
${ }^{*}$ These authors contributed equally to this work.

†yulin.chen@physics.ox.ac.uk
}

In transport measurements, the sample remains metallic down to $2 \mathrm{~K}$, and no magnetic order but an increase of saturated moment at low temperature $(\approx 4 \mathrm{~K})$ is observed from the magnetic susceptibility measurement. From ARPES measurements, comprehensive band structures are acquired, including the flat band predicted by first-principles calculation [12,1416], the large carrier density of which can enhance the TE performance at high temperatures. Using polarization dependent ARPES measurements, we find that the topmost valance bands mainly consist of the $\mathrm{Cu} d_{x z} / d_{y z}$ and $\mathrm{S} p_{x} / p_{y}$ orbitals. By providing a comprehensive reference of the electronic structure and related physical properties of $\mathrm{BiCuSO}$, our paper will assist the search for superconductivity, and promote the advancement of TE performance in $\mathrm{BiCuChO}$ single crystals.

\section{METHODS}

$\mathrm{BiCuSO}$ and $\mathrm{BiCuSeO}$ single crystals were grown by the chemical vapor transport method [24,25]. First, the polycrystalline samples were synthesized using a solid-state reaction method by heating the powders of Bi (Alfa Aesar, 99.999\%), $\mathrm{Bi}_{2} \mathrm{O}_{3}$ (Sinopharm Chemical Reagent 99.99\%), $\mathrm{Cu}$ (Sinopharm Chemical Reagent 99.99\%), and S (Alfa Aesar 99.5\%), or Se (Alfa Aesar 99.999\%) in a sealed evacuated quartz tube at about $700^{\circ} \mathrm{C}$ for seven days. Second, a certain amount of $\mathrm{BiCuSO}$ or $\mathrm{BiCuSeO}$ powder and transport agent $\mathrm{I}_{2}$ were mixed, loaded into the evacuated quartz ampoule, and then placed in a two-zone furnace with the temperature profile of $500-600{ }^{\circ} \mathrm{C}$ to grow the crystals. After over ten days, millimeter-sized single crystals with 
(a)

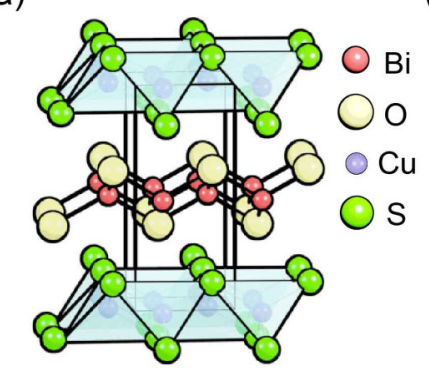

(b)

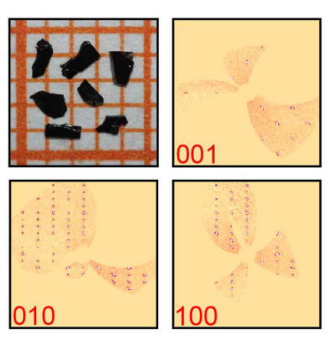

(c)

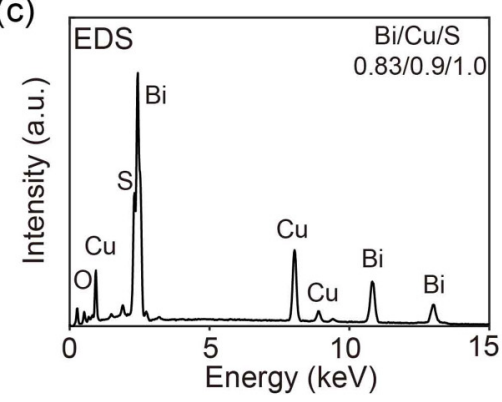

(d)

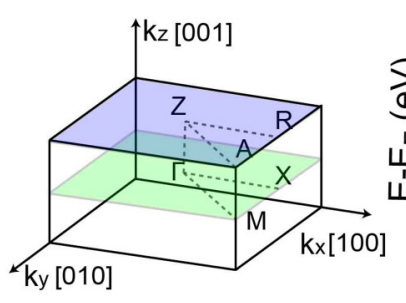

(e)

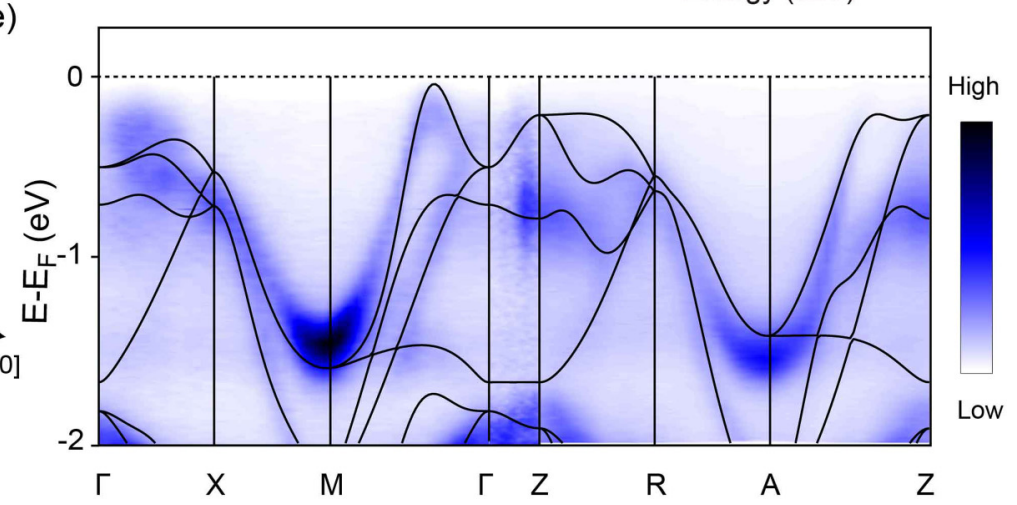

FIG. 1. Characterization of BiCuSO single crystals. (a) Schematic illustration of crystal structure, with CuS4 tetrahedral layers highlighted in light blue. (b) Photography and XRD patterns along different directions. (c) EDS results. (d) Brillouin zone labeled with high symmetry points. The $Z A R(Z)$ plane and $\Gamma M X(\Gamma)$ plane are marked in blue and green, respectively. (e) Electronic band dispersion along $\Gamma-X-M-\Gamma-$ $Z-R-A-Z$ overlapped with calculation.

metallic luster and flake shape were obtained. The crystal structure of the as-grown crystals was determined by singlecrystal x-ray-diffraction (XRD) measurement (Supernova, Oxford Instrument). The electrical transport measurements were performed with a $9 \mathrm{~T}$ physical properties measurement system (PPMS-9T, Quantum Design) by a standard fourprobe configuration. The characterizations of the magnetic properties were carried out in a magnetic property measurement system (MPMS3-SQUID, Quantum Design). The energy-dispersive x-ray spectroscopy (EDS) measurements were carried out with a scanning electron microscope (SEM, FEI-Quantum).

ARPES measurements on BiCuSO were performed at beamline I05 of Diamond Light Source and measurements on $\mathrm{BiCuSeO}$ were performed at beamline SIS of Swiss Light Source. The overall energy and angular resolutions are $\approx 10 \mathrm{meV}$ and $\approx 0.2^{\circ}$, respectively. The samples were cleaved in situ along the [001] direction and the ARPES measurements were carried out under ultrahigh vacuum of better than $1.3 \times 10^{-10}$ mbar at the temperature of $6 \mathrm{~K}$. The photon energies used in ARPES experiments on BiCuSO ranged from 120 to $210 \mathrm{eV}$ : fine maps and high symmetry cuts at the $\Gamma M X$ plane ( $\Gamma$ plane) and $Z A R$ plane $(Z$ plane) were measured at 182 and $164 \mathrm{eV}$, respectively. The Fermi level $\left(E_{F}\right)$ of BiCuSO was determined by the $E_{F}$ of gold measured in the same beamtime. For $\mathrm{BiCuSeO}$, the photon energy used in ARPES experiments was $186 \mathrm{eV}$ ( $\Gamma$ plane) and $154 \mathrm{eV}(Z$ plane).

The $a b$ initio calculations were performed within the framework of density functional theory as implemented in the Vienna Ab-Initio Simulation Package [26]. The HeydScuseria-Ernzerhof hybrid exchange-correlation function [27] and a $k$ mesh of $9 \times 9 \times 4$ were taken. Calculations without spin-orbit coupling are presented in this paper due to better consistency. Due to the stoichiometric structure used in the calculation, in BiCuSO, the calculated Fermi level locates $0.23 \mathrm{eV}$ higher than the valence band maximum (VBM); in $\mathrm{BiCuSeO}$, the calculated Fermi level locates $0.19 \mathrm{eV}$ higher than the VBM. In this paper, the calculated band structure of BiCuSO is shifted by $+0.20 \mathrm{eV}$ and renormalized by a factor of 1.01 ; that of $\mathrm{BiCuSeO}$ is shifted by $+0.19 \mathrm{eV}$.

\section{RESULTS AND DISCUSSION}

\section{A. Characterization of BiCuSO single crystals}

$\mathrm{BiCuSO}$ has the same crystal structure as $\mathrm{LaFeAsO}$ (space group $P 4 / \mathrm{nmm}$ ) [28], formed by the alternative stacking of insulating $\mathrm{BiO}_{4}$ layers and conductive $\mathrm{CuS}_{4}$ tetrahedral layers (highlighted by blue tetrahedra) [Fig. 1(a)]. Our singlecrystal samples appear as millimeter-sized thin flakes with black metallic luster [Fig. 1(b)]. The single-crystal XRD patterns confirm the high quality of our samples, where we extract the lattice constants of $a=3.87 \AA$ and $c=8.57 \AA$ [Fig. 1(b)]. The EDS measurement [Fig. 1(c)] presents characteristic peaks of all four elements, from which we find the $\mathrm{Bi} / \mathrm{Cu} / \mathrm{S}$ atomic ratio to be $0.83 / 0.9 / 1.0$. The established literature shows that Bi deficiency can function as hole doping which rigidly shifts the electronic structure, and $\mathrm{Cu}$ deficiency is predicted to induce magnetic order and superconductivity [14-16]. We notice that our samples have the same proportion of $\mathrm{Cu}$ deficiency as the previously reported $\mathrm{BiCuSO}$ samples that demonstrate superconductivity [13]. Figure 1(d) 
(a)

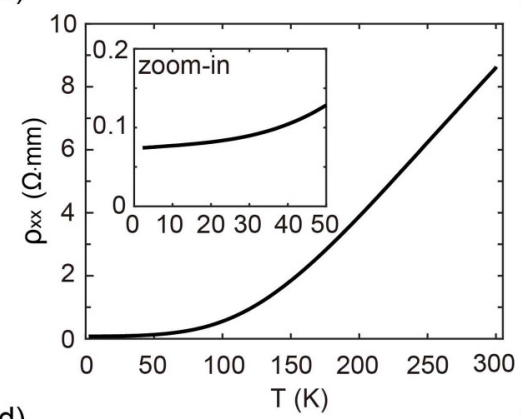

(d)

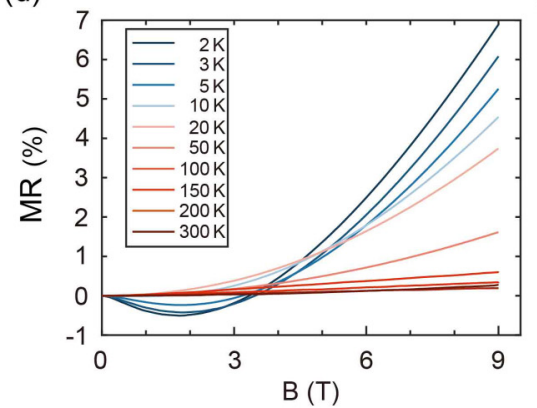

(b)

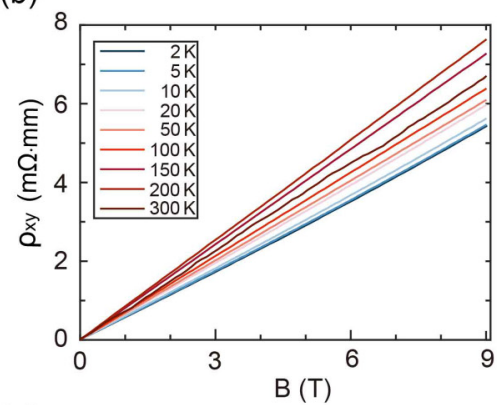

(e)

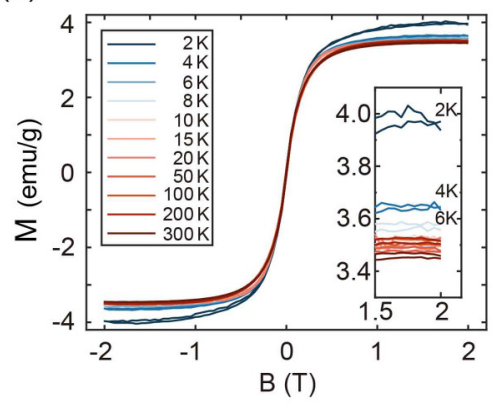

(c)

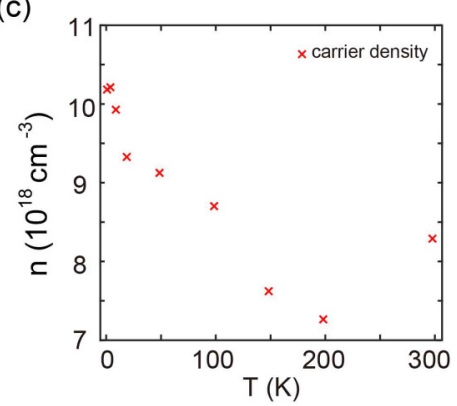

(f)

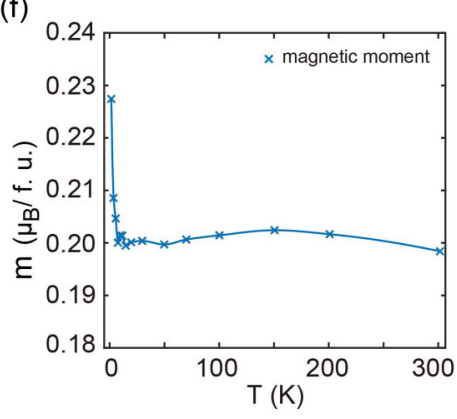

FIG. 2. Electrical and magnetic properties of BiCuSO. (a) Temperature-dependent resistivity curve, with a zoom-in at low temperature shown as the inset. (b) Hall resistivity curves at different temperatures. (c) Temperature-dependent carrier density extracted from (b). (d) Magnetoresistance (MR) curves at different temperatures. (e) Field-dependent magnetization at different temperatures, with zoom-in around $2 \mathrm{~T}$ shown as an inset. (f) Magnetic moments per formula unit (f.u.) at different temperatures extracted from the data in (e).

illustrates the Brillouin zone (BZ) of $\mathrm{BiCuSO}$, and the notation of high symmetry points when orbital characters are not considered. In the following content, we focus on the electronic structure in high symmetry planes, the $\Gamma$ plane (marked by green) and $Z$ plane (marked by blue). Figure 1(e) offers an overview of the electronic structure by presenting the band dispersion along $\Gamma-X-M-\Gamma-Z-R-A-Z$. We observe dispersive bands along $\Gamma-M$ and $Z-A$, which form multiple hole pockets near the VBM, in good consistency with the calculation. We also observe broad features along $\Gamma-X$ and $Z-R$, which can be attributed to the weakly dispersive bands in the calculation. This coexistence of light and heavy hole bands near the VBM is favorable for TE performance, as the light hole bands can contribute to good electrical conductivity while the heavy hole bands can maintain a large Seebeck coefficient, which endows $\mathrm{BiCuSO}$ with promising potential for thermoelectricity [29].

\section{B. Electrical properties of BiCuSO single crystals}

Electrical and magnetic properties of BiCuSO single crystals are presented in Fig. 2. Our samples exhibit the typical metallic behavior, showing a monotonically increasing resistivity $\left(\rho_{x x}\right)$ against temperature $(T)$ from 2 to $300 \mathrm{~K}$ [Fig. 2(a)]. The zoom-in view at low temperature confirms there is no superconducting transition down to $2 \mathrm{~K}$. The Hall measurements [Fig. 2(b)] show a linear increase of Hall resistivity $\left(\rho_{x y}\right)$ against magnetic field $(B)$ at all temperatures, indicating only a single type of charge carrier in BiCuSO crystals and that the dominant carriers are holes. Carrier densities [Fig. 2(c)] extracted from Hall coefficients have values around $10^{19} \mathrm{~cm}^{-3}$, an order of magnitude larger than that reported in polycrystalline samples [18-21,23], and show an increase of $\approx 40 \%$ from 200 to $2 \mathrm{~K}$. The magnetoresistance (MR) measurements [Fig. 2(d)] show negative MR below $10 \mathrm{~K}$ and no apparent variation above $100 \mathrm{~K}$. The metallic behavior in $\mathrm{BiCuSO}$ single crystals can be attributed to the $\mathrm{Bi}$ and $\mathrm{Cu}$ deficiencies, which introduce sufficient carriers to the degenerate phase and, meanwhile, result in weak localization at low temperature $[25,30]$.

\section{Magnetic properties of BiCuSO single crystals}

$\mathrm{Cu}$ in stoichiometric BiCuSO has an electronic configuration of $3 d^{10}$, which contributes zero magnetic moment according to Hund's rules [31]. Calculation shows that $10 \%$ $\mathrm{Cu}$ deficiency in $\mathrm{BiCuSO}$ is favorable for a ferromagnetic order, where $\mathrm{Cu}$ can contribute $\approx 0.12 \mu_{\mathrm{B}}$ per formula unit (f.u.) $[14,16]$. Karna et al. observed a saturated moment of $0.61 \mu_{\mathrm{B}} /$ f.u. at $3.7 \mathrm{~K}$ in $\mathrm{BiCu}_{0.94} \mathrm{SO}$ polycrystalline samples and claimed a canted ferromagnetic order, which evolves from an in plane antiferromagnetic order at $180 \mathrm{~K}[19,20]$. However, the magnetization loops [Fig. 2(e)] in our samples indicate no magnetic order down to $2 \mathrm{~K}$. Curiously, the zoom-in view around $2 \mathrm{~T}$ shows an increase of the saturated magnetization at low temperature. The saturated magnetic moment [Fig. 2(f)] extracted from Fig. 2(e) shows little variation $\left(\approx 0.20 \mu_{\mathrm{B}} /\right.$ f.u. $)$ when the temperature decreases from 300 to $8 \mathrm{~K}$, while it jumps to $\approx 0.23 \mu_{\mathrm{B}} / \mathrm{f} . \mathrm{u}$. $(+14 \%)$ when temperature further decreases to $2 \mathrm{~K}$. As our samples are flakelike and all the magnetic measurements were performed normal to the $a b$ plane, this sharp increase may result from the reorientation of the magnetic easy axis from in-plane to out-of-plane direction [19]. Another possible mechanism is 
(a)

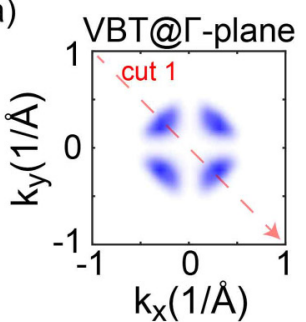

(c)

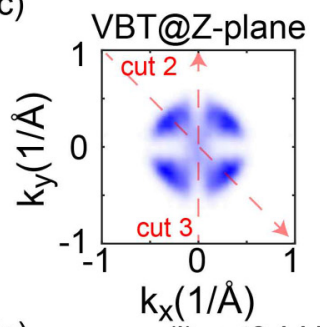

(e)

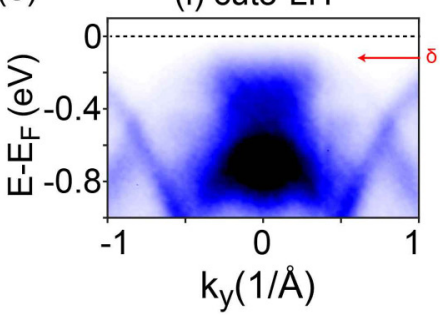

(d) (b) (i) cut1-LH
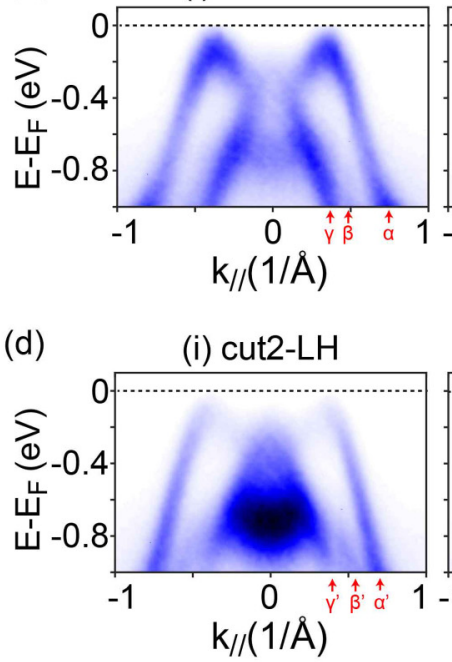

(ii) cut3-LV

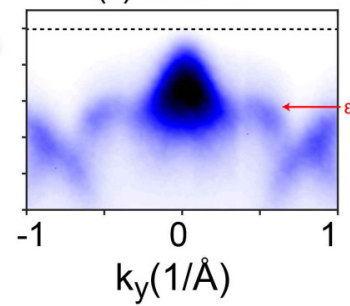

(ii) cut1-LV

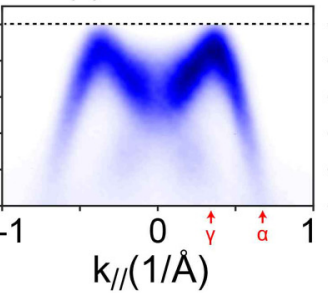

(ii) cut2-LV

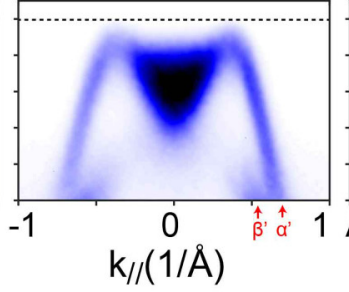

(iii) second derivative

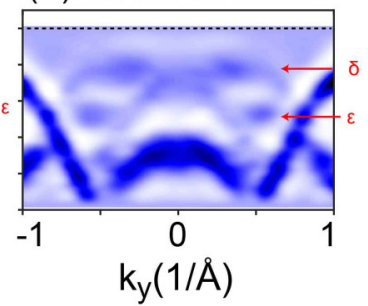

(iii) calculation

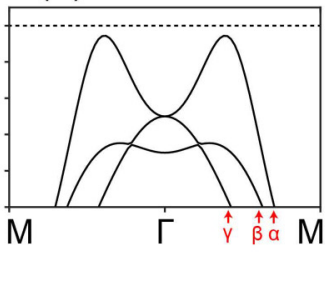

(iii) calculation

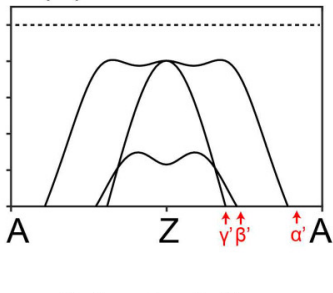

(iv) calculation

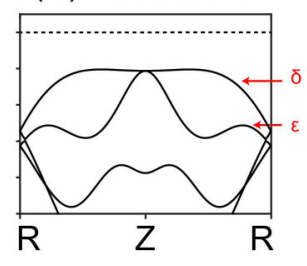

FIG. 3. Electronic structure of BiCuSO. (a) Equal energy contour at the valence band top (VBT) in the $\Gamma$ plane, measured in LH. (b) High symmetry cuts along $M-\Gamma-M$ measured in (i) LH, (ii) LV, and (iii) corresponding calculation. (c) Equal energy contour at the VBT in the $Z$ plane, measured in LH. (d) High symmetry cuts along $A-Z-A$ in (i) LH, (ii) LV, and (iii) corresponding calculation. (e) High symmetry cuts along $R-Z-R$ in (i) LH, (ii) LV, (iii) the second derivative of the LH results, and (iv) corresponding calculation. The equal energy contours are mirror symmetrized, and have an integration width of $50 \mathrm{meV}$.

the change of $\mathrm{Cu} 3 d$ electronic configuration (chemical valence), which can also lead to the increase of the saturated moment [32]. Detailed investigation on the magnetic order of the BiCuSO single crystal is needed for clarifying this phenomenon.

\section{Electronic structure of $\mathrm{BiCuSO}$}

Figure 3 presents the electronic structure of BiCuSO. The equal energy contour at the valence band top (VBT) in the $\Gamma$ plane [Fig. 3(a)] shows quadruple segments located between $\Gamma$ and $M$. In the high symmetry cut along $M-\Gamma-M$ measured using linear-horizontally $(\mathrm{LH})$ polarized photons [Fig. 3(b-i)], we observe the three bands predicted by the calculation [Fig. 3(b-iii)]: the band $\alpha$ that forms the VBM the center of which locates at $k_{\|}= \pm 0.35 \AA^{-1}$; the hole band $\gamma$ the maximum of which locates at $\Gamma \sim 0.24 \mathrm{eV}$ below $E_{F}$; and the band $\beta$ that appears as broad features with weak intensity. By fitting the VBM to a parabola, we extract an effective mass of about $-0.56 m_{e}$ (Fig. 4). In the high symmetry cut measured using linear-vertically (LV) polarized photons [Fig. 3(b-ii)], we observe intensive band $\alpha$ around the VBM while diminishing bands $\beta$ and $\gamma$ because of orbital selection. The equal energy contour at VBT in the $Z$ plane [Fig. 3(c)] presents a weak feature at the center apart from the quadruple segments, indicating the presence of additional electronic structure near $E_{F}$. In the LH cut along A-Z-A [Fig. 3(d-i)], we observe similar band dispersion as along $M-\Gamma-M$, featured by the dispersive bands $\alpha^{\prime}$ and $\gamma^{\prime}$. In the LV cut along $A-Z-A$ [Fig. 3(d-ii)], we determine the maximum of band $\alpha^{\prime}$ at $k_{\|}=$ $\pm 0.38 \AA^{-1}$ and extract the effective mass of $-0.63 m_{e}$ (Fig. 4). The hole band at $Z$ cannot be clearly identified due to the intensive triangular feature, which results from the resonance of S $2 p$ orbitals [33].

It is notable that calculations predicted flat bands along $R-Z-R$ near the VBM $[12,14-16,21]$, which could be the origin of the high $Z T$ value in the hole-doped BiCuSO. First, this flat band contributes to a local extremum in the density
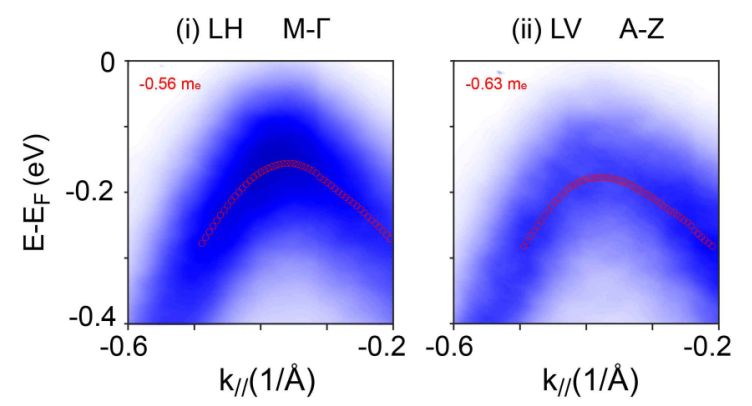

FIG. 4. Zoom-in view around the valence band top. (i) Zoom-in view of Fig. 3(b-i). (ii) Zoom-in view of Fig. 3(d-ii). The red circles show the peak positions of the energy distribution curves, which are fitted with parabolic curves to obtain the effective masses. 
(a)

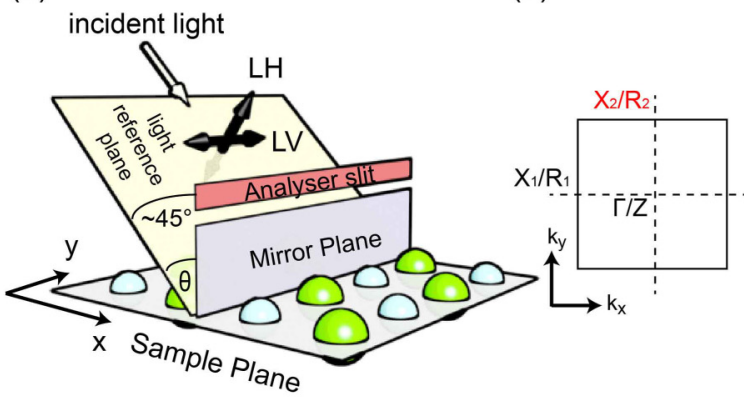

(c)

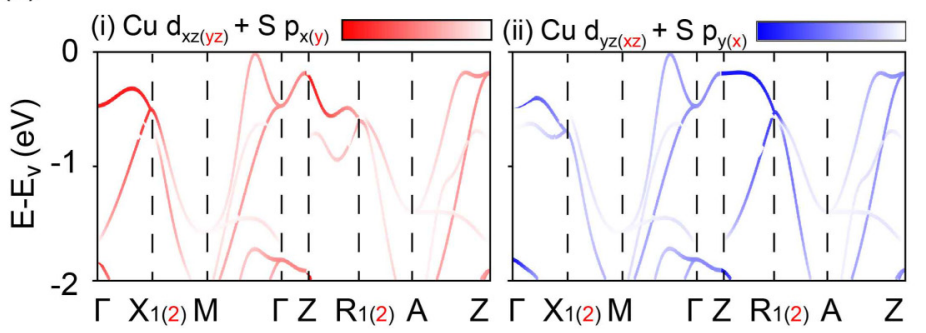

VBT@Z-plane

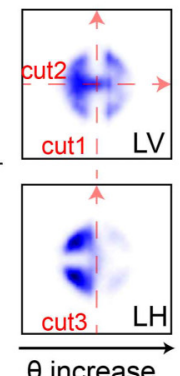

$\theta$ increase

(d)

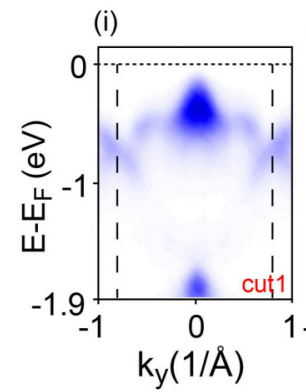

(ii)

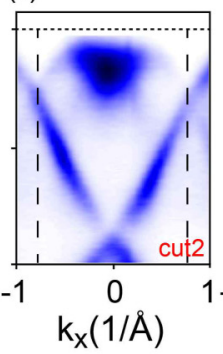

(e)

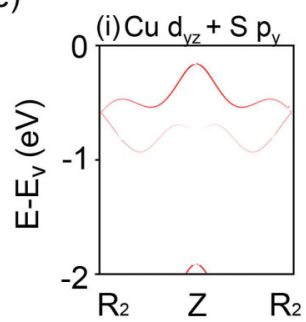

(iii)

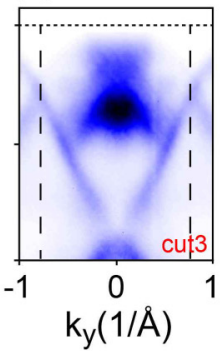

FIG. 5. Orbital characters of the valence band in BiCuSO. (a) Schematic illustration of the ARPES experimental geometry. (b) Left panel: Brillouin zone (BZ) and notation used in the calculation of orbital character. Right panel: Equal energy contours at VBT in the $Z$ plane measured in LV and LH. The dashed lines in the left panel are used for locating the high symmetry points. (c) Calculated orbital weights of (i) $\mathrm{Cu} d_{x z}+\mathrm{S} p_{x}$ and (ii) $\mathrm{Cu} d_{y z}+\mathrm{S} p_{y}$ along the horizontal direction (black notation), or orbital weights of (i) $\mathrm{Cu} d_{y z}+\mathrm{S} p_{y}$ and (ii) $\mathrm{Cu} d_{x z}+\mathrm{S} p_{x}$ along the vertical direction (red notation). (d) High symmetry cuts along $R-Z-R$ measured in (i) LV and (iii) LH, and (ii) cut along $R-Z-R$ obtained from the map data. The vertical dashed lines show the boundary of BZ. (e) Simulation results following the photoemission selection rules.

of states (DOS) [14-16], which could account for the dramatic increase of carrier density and the enhancement of electrical conductivity in the p-type samples [9]. Second, according to the Pisarenko relationship [34], charge carriers with large effective mass are necessary for the large Seebeck coefficient. According to both our experimental data and the calculation, the valence band top along the [110] direction ( $\Gamma-M$ and $Z-A$ ) shows dispersive bands, which contributes a hole carrier with small effective masses (Fig. 4) and is favorable for the electrical conductivity. The heavy carriers are most likely contributed from the heavy hole bands along the [100] direction $(\Gamma-X$ and $Z-R)$, or the fine structures around $Z[10,12]$.

Our calculation [Fig. 3(e-iv)] reproduces the flat band (band $\delta$ ) along $R-Z-R$ and the hole band at $Z$ (band $\epsilon$ ). In the LH cut along $X-\Gamma-X$ [Fig. 3(e-i)], we observe flat features in the center, and weak intensity at both sides [also observed in Fig. 5(d-ii)] at $\approx 0.2 \mathrm{eV}$ below $E_{F}$. These features are clearly presented by the second derivative along the energy axis [Fig. 3(e-iii)], showing the weakly dispersive band ranging from $k_{y}=-0.4$ to $0.4 \AA^{-1}$ (band $\delta$ ) and the hole band at $Z$ (band $\epsilon$ ), which are qualitatively consistent with the calculation. In the LV cut along $X-\Gamma-X$ [Fig. 3(e-ii)], we observe the band $\epsilon$ with strong intensity around the top. From the maximum of the hole band at $Z$, we estimate a binding energy of $\approx 150 \mathrm{meV}$ the flat band, which is significantly larger than $k_{B} T$ at low temperature $\left(k_{B}\right.$ is the Boltzmann constant). We therefore conclude that the electrical properties of hole-doped $\mathrm{BiCuSO}$ at low temperature are dominated by the quadruple hole bands between $\Gamma(Z)$ and $M(A)$. However, in the high temperature range $(T>1000 \mathrm{~K})$, this flat band will be involved in the TE process, and substantially contribute to the Seebeck coefficient as well as the carrier density [3,12].

\section{E. Orbital characters of BiCuSO}

Analysis of the polarization dependence of the ARPES results reveals the orbital characters of the valence band (Fig. 5). The experimental geometry for the ARPES measurement is illustrated in Fig. 5(a). The sample mirror plane is aligned coincident with the direction of the analyzer slit while measuring high symmetry cuts along $R-Z-R$ (cuts 1 and 3 ). In this geometry, the LV cut is supposed to show zero intensity of $\mathrm{Cu} 3 d_{x z}, 3 d_{x y}$, and S $3 p_{x}$ orbitals, and the LH cut is supposed to show zero intensity of $\mathrm{Cu} 3 d_{y z}, 3 d_{x^{2}-y^{2}}$, and $\mathrm{S} 3 p_{y}$ while presenting some intensity from the out-of-plane orbital components $\left(\mathrm{Cu} d_{z^{2}}\right.$ and $\left.\mathrm{S} p_{z}\right)$ [35,36].

We find the electronic structure of $\mathrm{BiCuSO}$ no longer follows the fourfold symmetry but only mirror symmetry when orbital characters are considered, and thus redefine the BZ as the left panel of Fig. 5(b). This reduced symmetry is directly presented by the original data of LV and LH equal energy contours at VBT [Fig. 5(b), right panels]. The calculated orbital weights show that the valence band top is mainly contributed from the hybridization of $\mathrm{Cu} 3 d_{x z}$ and $\mathrm{S} 3 p_{x}, \mathrm{Cu} 3 d_{y z}$, and $\mathrm{S} 3 p_{y}$ orbitals [Fig. 5(c)], indicating the formation of a quasi-one-dimensional electronic structure in real space (Fig. 6), which is favorable for the TE performance [37-40]. We also notice some contribution from the $\mathrm{Bi}$ orbitals at the topmost band along $\Gamma-X$ and $\Gamma-Z$ (Fig. 7), the maximum percentage of which is $10.76 \%$ at 


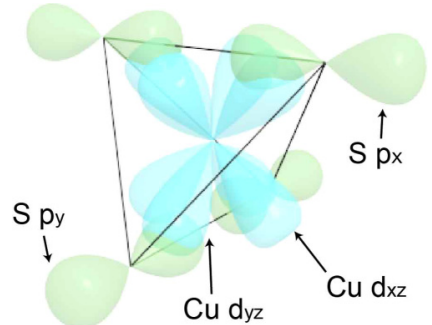

FIG. 6. Illustration of the quasi-one-dimensional conductive chain in $\mathrm{CuS}_{4}$ tetrahedron.

$\Gamma$, which is smaller than that reported in $\mathrm{BiCuSeO}(\approx 20 \%)$ [37]. The flat band along $Z-R_{1}$ is dominantly contributed from $\mathrm{Cu} d_{y z}+\mathrm{S} p_{y}$ orbitals while that along $Z-R_{2}$ is dominantly contributed from $\mathrm{Cu} d_{x z}+\mathrm{S} p_{x}$ orbitals. This nonequivalence in orbital weight is demonstrated by comparing the cuts along nominally equivalent directions [Fig. 5(d)]. Both cuts 1 and 2 [Figs. 5(d-i) and 5(d-ii)] follow the same selection rule and present the intensity from $\mathrm{Cu} d_{y z}+\mathrm{S} p_{y}$ orbitals as predicted by simulation [Figs. 5(e-i) and 5(e-ii)]. We attribute the intensity below the flat band [Fig. 5(d-ii)] to the resonance induced broadening [33]. Compared with cut 2, cut 3 shows stronger intensity at around $-0.8 \mathrm{eV}$, which is contributed from the $\mathrm{Cu} d_{z^{2}}$ and $\mathrm{S} p_{z}$ electrons excited by LH photons [Fig. 5(e-iii)].

\section{F. Comparison with $\mathrm{BiCuSeO}$}

To understand the communal origin of promising TE performance in the $\mathrm{BiCuChO}$ family, we further investigate the electronic structure of $\mathrm{BiCuSeO}$ (Fig. 8). Figure 8(a) presents an overview of the electronic structure of $\mathrm{BiCuSeO}$, featured by the coexistence of light and heavy hole bands near the VBT. The Fermi surface of BiCuSeO [Fig. 8(b-i)] presents a cruciform surrounded by a square cage, obviously different from the VBT of BiCuSO [Fig. 3(c)]. The cut along $A-Z-A$ [Fig. 8(b-ii)] presents a clear $E_{F}$ crossing from the outside bands, showing the VBM is above $E_{F}$. The cut along $R-Z-R$ [Figs. 8(b-iii) and $8(\mathrm{~b}-\mathrm{iv})$ ] presents a hole pocket capped by the broad intensity in the vicinity of $E_{F}$, which can be attributed to the weakly dispersive band in the calculation along $Z$-R [Fig. 8(a)].

Our observation confirms the overall similarity in the valence band structure in $\mathrm{BiCuChO}$, which is rooted in the similarity of crystal structure [12]. Similar to BiCuSO, the topmost bands in $\mathrm{BiCuSeO}$ are strongly dispersive along the [110] direction while weakly dispersive along the [100] direction, indicating the observed "flat" band along $Z-R$ could serve as the origin for the large Seebeck coefficient required by the Pisarenko relation [34]. Additionally, this flat band can also contribute to a large DOS at $E_{F}$, favorable for the increase of carrier density and the enhancement of electrical conductivity in the hole-doping process [3-7,10,11,25].

Despite the common points, our calculation also presents divergences in the VBT of $\mathrm{BiCuChO}$, particularly around $Z$. Unlike BiCuSO [Fig. 1(e)], the Z-centered hole pocket in $\mathrm{BiCuSeO}$ is obviously higher than the nearby hole bands; the weakly dispersive band along $Z-R$ also shows subtle differences. We notice that these fine structures near the VBT contribute to complex thermoelectric behavior in $\mathrm{BiCuSeO}$
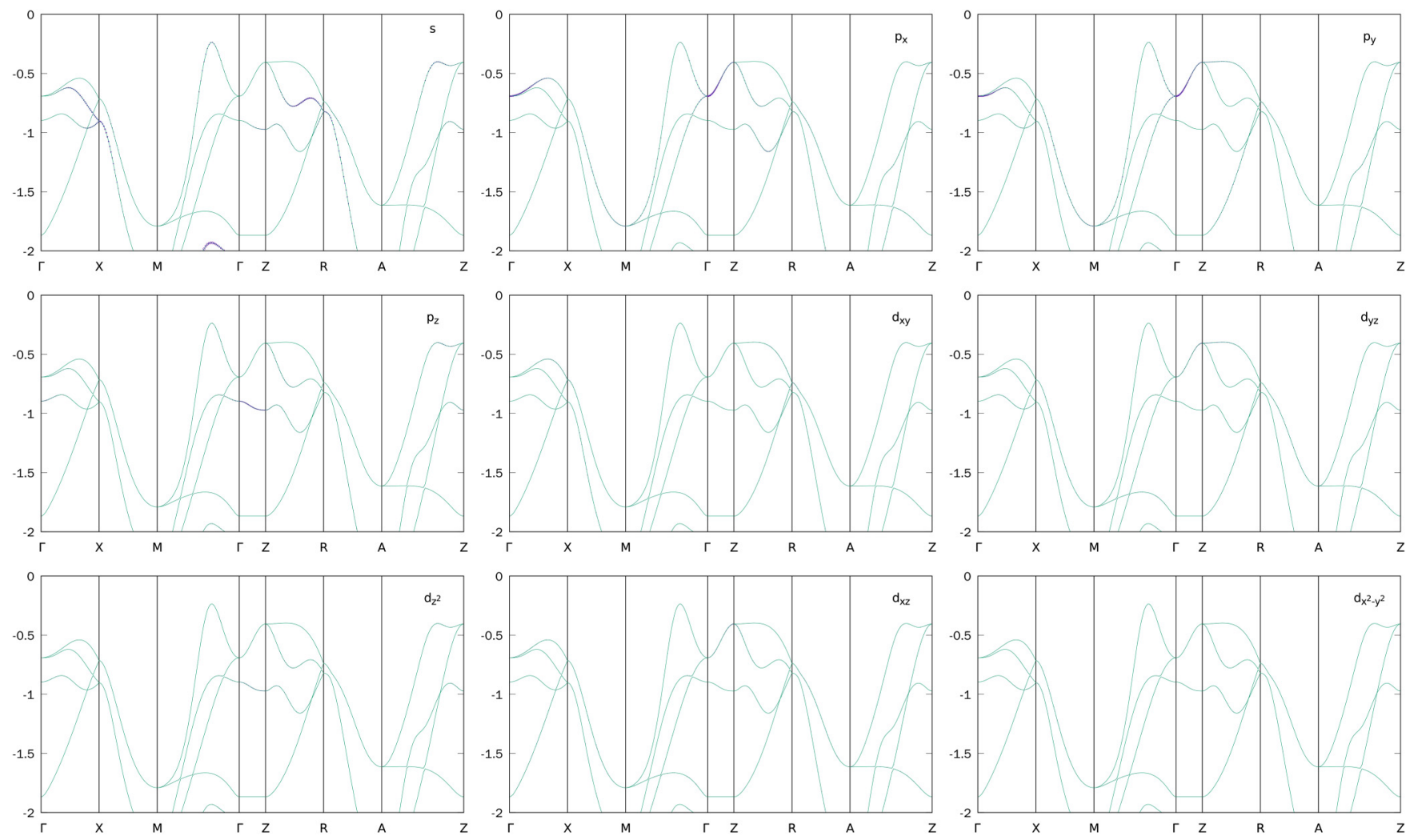

FIG. 7. Orbital weight of $\mathrm{Bi}$. The size of the purple circle is proportional to the contribution from Bi orbitals. 
(a)

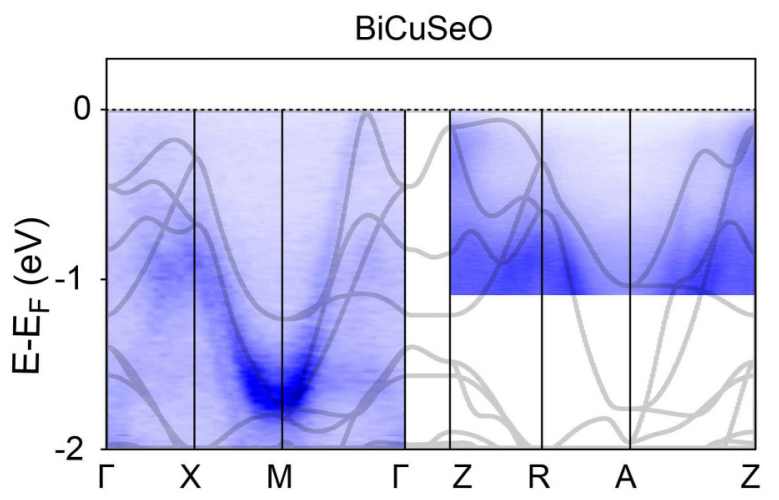

(b)
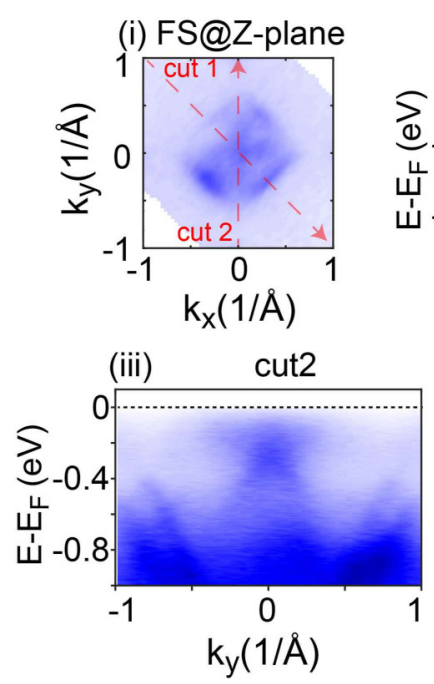

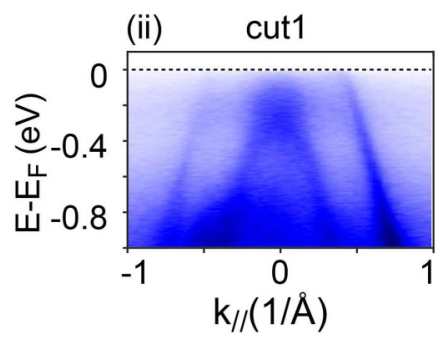

(iv) second derivative

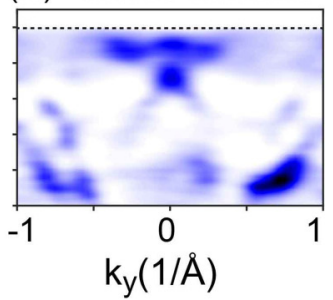

FIG. 8. Electronic structure of BiCuSeO. (a) Electronic band dispersion along $\Gamma-X-M-\Gamma-Z-R-A-Z$ overlapped with calculation. (b-i) Fermi surface map in the $Z$ plane. (ii) Cut along $A-Z-A$. (iii) Cut along $R-Z-R$ and (iv) corresponding second derivative. The data in the $Z$ plane were measured in circular-left (CL) polarization at $154 \mathrm{eV}$. The data in the $\Gamma$ plane were measured in $\mathrm{LH}$ polarization at $186 \mathrm{eV}$.
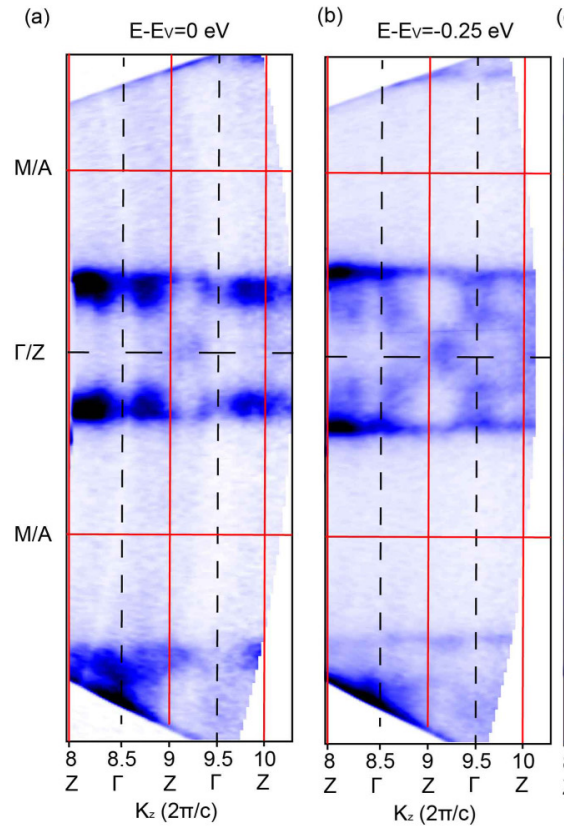

(c) $\quad E-E v=-0.5 \mathrm{eV}$

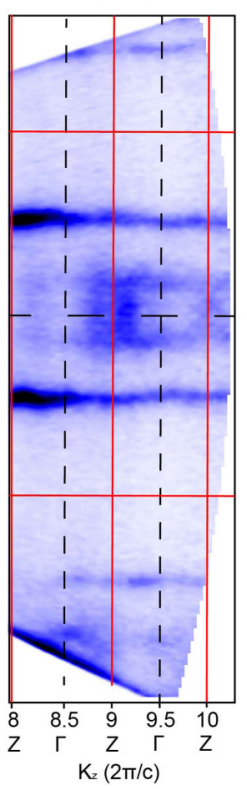

(d)

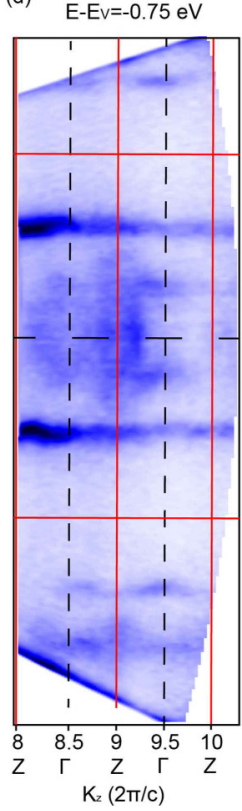

(e)

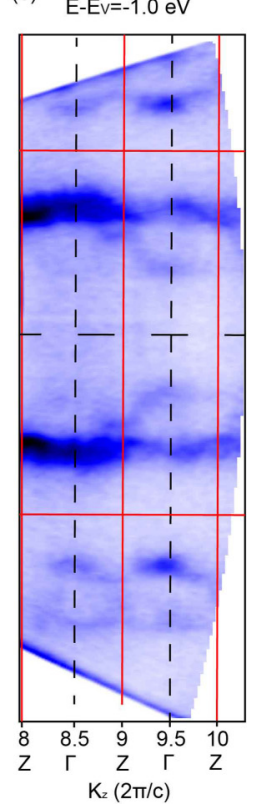

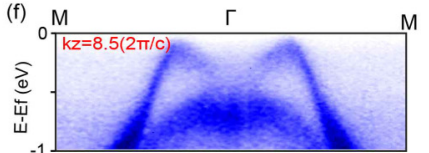

(g)
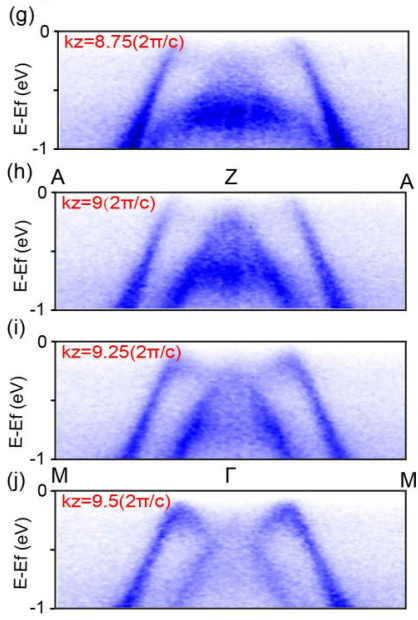

FIG. 9. Electronic structure of BiCuSO along $k_{z}$. (a)-(e) Equal energy contours of $k_{z}$-dependent data, overlapped with the Brillouin zones along the corresponding direction. (f)-(j) Cuts along the [110] direction at the different $k_{z}$ positions.

(a)

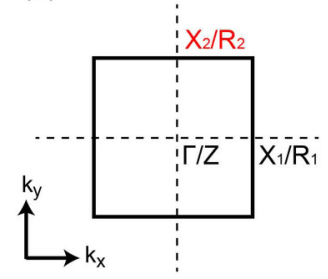

(b) $\mathrm{Cud} \mathrm{d}_{x^{2}-y^{2}}$

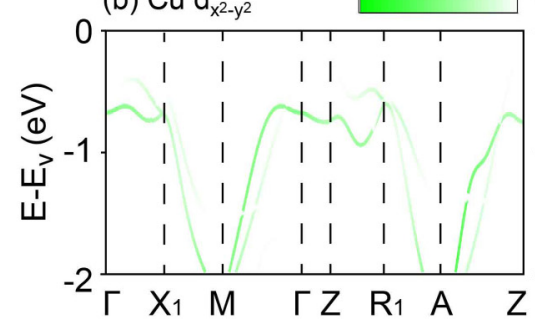

(c) $\mathrm{Cud}_{\mathrm{z}^{2}}+S \mathrm{p}_{\mathrm{z}}$

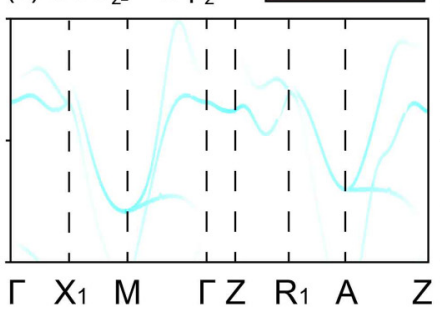

(d) $\mathrm{Cud}_{\mathrm{xy}}$

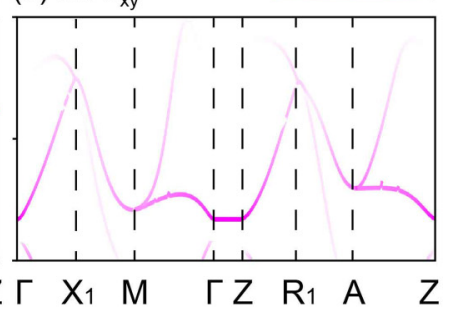

FIG. 10. Supplementary calculation of the orbital characters in BiCuSO. (a) Brillouin zone and notation used in the calculation of orbital character. (b)-(d) Calculated orbital weights of (b) $\mathrm{Cu} d_{x^{2}-y^{2}}$, (c) $\mathrm{Cu} d_{z^{2}}+\mathrm{S} p_{z}$, and (d) $\mathrm{Cu} d_{x y}$. 
[10]. Unfortunately, these fine structures were unobservable in the ARPES measurements due to the heavily hole-doped samples. A detailed investigation on the electronic structure of $\mathrm{BiCuSeO}$ is needed for understanding the divergence in $\mathrm{TE}$ performance of the $\mathrm{BiCuChO}$ family.

\section{SUMMARY}

In summary, we investigate the transport properties and the electronic structure of hole-doped BiCuSO single crystals [Figs. 3, 8 and 9]. Our samples present metallic transport behaviors without superconducting transition down to $2 \mathrm{~K}$. The magnetization loops at different temperatures present no magnetic order, while the increase of saturated magnetic moment at $4 \mathrm{~K}$ indicates possible reorientation of the easy axis. Our ARPES measurements directly visualize the valence band structure of $\mathrm{BiCuSO}$ and $\mathrm{BiCuSeO}$, especially the weakly dispersive bands along $Z-R$. These flat bands could account for the enhancement of electrical conductivity in the hole-doping process, and contribute hole carriers with a large effective mass that are favorable for a large Seebeck coefficient. We also reveal the orbital characters of the VBT in BiCuSO, which mainly consists of $\mathrm{Cu} 3 d_{x z}$ and $\mathrm{S} 3 p_{x}, \mathrm{Cu} 3 d_{y z}$, and S $3 p_{y}$ orbitals [Figs. 5 and 10].

\section{ACKNOWLEDGMENTS}

We thank J.-C. Lin for the helpful discussion on the transport data, and we thank P. Dudin, M. Shi, S. Stolz, and J. Krieger for the support in the ARPES experiment. The ARPES experiments are supported by Diamond Light Source under Proposals No. SI20617-1 and No. SI25135-1, and by Swiss Light Source under Proposal No. 20181102. D.P. appreciates the support from China Scholarship Council. Y.W.L. acknowledges the support from International Postdoctoral Exchange Fellowship Program (Talent-Introduction Program, Grant No. YJ20200126). Y.-Y.L. and Y.B.C. acknowledge the financial support from the National Natural Science Foundation of China (Grants No. 51902152 and No. 11874210). Y.Y.Y.X. and G.L. acknowledge the financial support from National Key Research and Development Program of China (Grant No. 2017YFE0131300), the Strategic Priority Research Program of Chinese Academy of Sciences (Grant No. XDA18010000), and Science and Technology Commission of Shanghai Municipality Support Program (Project No. 20DZ1100605).
[1] R. Viennois, P. Hermet, M. Beaudhuin, J. L. Bantignies, D. Maurin, S. Pailhès, M. T. Fernandez-Diaz, M. M. Koza, C. Barreteau, N. Dragoe et al., J. Phys. Chem. C 123, 16046 (2019).

[2] S. Kumar and U. Schwingenschlogl, Phys. Chem. Chem. Phys. 18, 19158 (2016).

[3] L.-D. Zhao, J. He, D. Berardan, Y. Lin, J.-F. Li, C.-W. Nan, and N. Dragoe, Energy Environ. Sci. 7, 2900 (2014).

[4] Y. Liu, L. D. Zhao, Y. Liu, J. Lan, W. Xu, F. Li, B. P. Zhang, D. Berardan, N. Dragoe, Y. H. Lin et al., J. Am. Chem. Soc. 133, 20112 (2011).

[5] Y. Liu, L.-D. Zhao, Y. Zhu, Y. Liu, F. Li, M. Yu, D.-B. Liu, W. Xu, Y.-H. Lin, and C.-W. Nan, Adv. Energy Mater. 6, 1502423 (2016)

[6] J. Li, J. Sui, C. Barreteau, D. Berardan, N. Dragoe, W. Cai, Y. Pei, and L.-D. Zhao, J. Alloys Compd. 551, 649 (2013).

[7] J. L. Lan, Y. C. Liu, B. Zhan, Y. H. Lin, B. Zhang, X. Yuan, W. Zhang, W. Xu, and C. W. Nan, Adv. Mater. 25, 5086 (2013).

[8] C. Barreteau, D. Bérardan, E. Amzallag, L. Zhao, and N. Dragoe, Chem. Mater. 24, 3168 (2012).

[9] J.-B. Labégorre, R. Al Rahal Al Orabi, A. Virfeu, J. Gamon, P. Barboux, L. Pautrot-d'Alençon, T. Le Mercier, D. Berthebaud, A. Maignan, and E. Guilmeau, Chem. Mater. 30, 1085 (2018).

[10] G. K. Ren, S. Wang, Z. Zhou, X. Li, J. Yang, W. Zhang, Y. H. Lin, J. Yang, and C. W. Nan, Nat. Commun. 10, 2814 (2019).

[11] Y.-L. Pei, J. He, J.-F. Li, F. Li, Q. Liu, W. Pan, C. Barreteau, D. Berardan, N. Dragoe, and L.-D. Zhao, NPG Asia Mater. 5, e47 (2013).

[12] D. Zou, S. Xie, Y. Liu, J. Lin, and J. Li, J. Mater. Chem. A 1, 8888 (2013).

[13] A. Ubaldini, E. Giannini, C. Senatore, and D. van der Marel, Physica C 470, S356 (2010).
[14] I. I. Mazin, Phys. Rev. B 81, 140508(R) (2010).

[15] I. R. Shein and A. L. Ivanovskii, Solid State Commun. 150, 640 (2010).

[16] L. Ortenzi, S. Biermann, O. K. Andersen, I. I. Mazin, and L. Boeri, Phys. Rev. B 83, 100505(R) (2011).

[17] A. Pal, H. Kishan, and V. P. S. Awana, J. Supercond. Novel Magn. 23, 301 (2010).

[18] D. Berthebaud, E. Guilmeau, O. I. Lebedev, A. Maignan, J. Gamon, and P. Barboux, J. Solid State Chem. 237, 292 (2016).

[19] S. K. Karna, R. Sankar, C.-M. Wu, C.-W. Wang, D. Hsu, C.-J. Wang, F.-C. Chou, and W.-H. Li, J. Phys. Soc. Jpn. 80, 011 (2011).

[20] S. K. Karna, C. W. Wang, C. M. Wu, C. K. Hsu, D. Hsu, C. J. Wang, W. H. Li, R. Sankar, and F. C. Chou, J. Phys.: Condens. Matter 24, 266004 (2012).

[21] D. Berardan, J. Li, E. Amzallag, S. Mitra, J. Sui, W. Cai, and N. Dragoe, Materials (Basel) 8, 1043 (2015).

[22] H. Zhu, T. Su, H. Li, C. Pu, D. Zhou, P. Zhu, and X. Wang, J. Eur. Ceram. Soc. 37, 1541 (2017).

[23] J. Gamon, S. Haller, E. Guilmeau, A. Maignan, T. Le Mercier, and P. Barboux, J. Solid State Chem. 263, 157 (2018).

[24] S.-S. Chen, Y.-C. Luo, Y.-Y. Zhang, S.-T. Dong, Y.-Y. Lv, Y.-S. Cui, S.-H. Yao, J. Zhou, and Y. B. Chen, J. Appl. Phys. 126, 055108 (2019).

[25] L. Xu, Y.-C. Luo, Y.-Y. Lv, Y.-Y. Zhang, S. Han, S.-H. Yao, J. Zhou, Y. B. Chen, and Y.-F. Chen, Cryst. Eng. Commun. 23 273 (2021).

[26] G. Kresse and J. Furthmüller, Phys. Rev. B 54, 11169 (1996).

[27] J. Heyd and G. E. Scuseria, J. Chem. Phys. 120, 7274 (2004).

[28] L. X. Yang, B. P. Xie, Y. Zhang, C. He, Q. Q. Ge, X. F. Wang, X. H. Chen, M. Arita, J. Jiang, K. Shimada et al., Phys. Rev. B 82, 104519 (2010). 
[29] K. Kuroki and R. Arita, J. Phys. Soc. Jpn. 76, 083707 (2007).

[30] S. Kawaji, Surf. Sci. 170, 682 (1986).

[31] W. Kutzelnigg and J. D. Morgan III, Z. Phys. D 36, 197 (1996).

[32] C. M. Varma, Rev. Mod. Phys 48, 219 (1976).

[33] M. Cardona and E. L. Ley, in Photoemission in Solids I: General Principles, 1st ed. (Springer-Verlag, Berlin, 1978), pp. 105134.

[34] G. J. Snyder and E. S. Toberer, Nat. Mater. 7, 105 (2008).

[35] S. Moser, J. Electron Spectrosc. Relat. Phenom. 214, 29 (2017).
[36] H. Lohani, T. Hazra, A. Ribak, Y. Nitzav, H. Fu, B. Yan, M. Randeria, and A. Kanigel, Phys. Rev. B 101, 245146 (2020).

[37] M. Ochi, H. Mori, D. Kato, H. Usui, and K. Kuroki, Phys. Rev. Mater. 2, 085401 (2018).

[38] C. Lee, T.-H. An, E. E. Gordon, H. S. Ji, C. Park, J.-H. Shim, Y. S. Lim, and M.-H. Whangbo, Chem. Mater. 29, 2348 (2017).

[39] M. S. Dresselhaus, G. Chen, M. Y. Tang, R. G. Yang, H. Lee, D. Z. Wang, Z. F. Ren, J. P. Fleurial, and P. Gogna, Adv. Mater. 19, 1043 (2007).

[40] L. D. Hicks and M. S. Dresselhaus, Phys. Rev. B 47, 16631 (1993). 